\title{
Lattice Boltzmann Method to Study Hydrodynamic Properties of 2D Fractal Aggregates
}

\author{
Hung P. Nguyen ${ }^{1}$, Bastien Chopard ${ }^{1}$ and Serge Stoll ${ }^{2}$ \\ 1 Department of Computer Science, \\ University of Geneva, Switzerland \\ 2 Chimie Analytique et Biophysico-chimie de l'Environnement (CABE) \\ University of Geneva, Switzerland
}

\begin{abstract}
The Lattice Boltzmann approach for fluid dynamics is used to compute the drag force on various types of obstacles in two-dimensional flows. We study the finite size effects for the drag force on a cylinder and propose a scaling law. Then we consider the hydrodynamic behavior of fractal objects and compare the drag force they experience with that of simpler geometries. Simulation results show that, in $2 \mathrm{D}$, the outer shape of the fractal plays the main role to determine the drag whereas the internal structure has little influence.
\end{abstract}

\section{Introduction}

Hydrodynamic properties of fractal aggregates have attracted attention of scientists for several decades. Investigations of the hydrodynamic properties of fractals are related to many aspects, from theoretical analysis of fractal aggregates suspended in viscous fluid to analytical and numerical approximations of Stokes' equation and transport properties in sedimentation, filtration and electrophoresis processes. Literature in this field is mainly concentrated on the calculation of the drag force $F_{D}$ acting on fractals in viscous fluid, determination of the settling velocities $V$, hydrodynamic radius $R_{h}$ of fractals $\ldots$ and their connections to fluid viscosity $\mu$, fractal dimension $D_{f}$ and other relevant factors.

In early researches, the Kirkwood Riseman theory that describes drag force on the elementary particles of a fractal in fluid by using interaction tensors between mutual interactions was used [1]. Using this approach P. Meakin [2], W. Hess, H.L. Frisch [3] investigated the ratio of the hydrodynamic radius to the gyration radius of fractal aggregates $R_{h} / R_{g}$. Other researches from P. Adler [4], B.Cichocki [5] concentrated on the determination of the drag force exerted by the surrounding fluid on a translating aggregate by numerically solving Stokes equation. However, very few work have been done for determining drag using numerical simulations. To the best of our knowledge, there are two contributions to this aspect, one is from P.Adler by solving Stokes equation using finite elements method [6] and the other is from A. Adrover and A.Giona using Lattice Boltzmann method [7]. 
The paper is organized as follow: first, we briefly discuss the Lattice Boltzmann (LB) method for simulation of fluid dynamics. Then the fluid motion past a cylinder is investigated in terms of channel width and discretization effects. Finally, the drag force exerted on a 2D fractal aggregate and fluid velocity is investigated and the relationship between the fluid velocity and drag force established.

\section{Lattice Boltzmann Approach for Numerical Simulation of Fluid Flow}

Lattice Boltzmann (LB) models are rather new numerical techniques aimed at modeling a physical system in terms of the dynamics of fictitious particles $[8,9]$. The main idea of this approach is to model the physical reality at a mesoscopic level: the generic features of microscopic processes can be expressed through simple rules, from which the desired macroscopic behavior emerges as a collective effect of the interactions between the many elementary components. In a LB model, the fluid is described by density distribution functions $f_{i}(\mathbf{r}, t)$ giving the probability that a fictitious fluid particle with velocity $\mathbf{v}_{i}$ enters the lattice site $\mathbf{r}$ at discrete time $t$. The admissible velocities $\mathbf{v}_{i}$ are dependent on the lattice topology. Usually, $i$ runs between 0 and $z$, where $z$ is the lattice coordination number (i.e. the number of lattice links). By convention $\mathbf{v}_{0}=0$ and $f_{0}$ represents the density distribution of particles at rest. The fluid density $\rho(\mathbf{r}, t)$ and fluid velocity $\mathbf{u}(\mathbf{r}, t)$ are defined as

$$
\rho=\sum_{i=0}^{z} f_{i} \quad \rho \mathbf{u}=\sum_{i=0}^{z} f_{i} \mathbf{v}_{i}
$$

The dynamics we consider for the $f_{i}$ 's is given by the so-called BGK model [9, $8]$

$$
f_{i}\left(\mathbf{r}+\Delta t \mathbf{v}_{i}, t+\Delta t\right)=\frac{1}{\tau} f_{i}^{(0)}(\mathbf{r}, t)+\left(1-\frac{1}{\tau}\right) f_{i}(\mathbf{r}, t)+\frac{\Delta t}{c_{s}^{2}} t_{i} \mathbf{F} \cdot \mathbf{v}_{i}
$$

where $\Delta t$ is the time step of the simulation, $\tau$ the so-called relaxation time and $\mathbf{F}$ an external force. The functions $f_{i}^{(0)}$ are called the local equilibrium distribution. They depend only on the fluid density $\rho$ and fluid velocity $\mathbf{u}$ in a suitable way. It can be shown (see for instance $[9,8,10]$ ) that equation (2) reproduces a hydrodynamical behavior if the local equilibrium functions are chosen as follows (Greek indices label the spatial coordinates and we use the Einstein convention of summation over repeated Greek indices)

$$
\begin{aligned}
& f_{i}^{(0)}=\rho t_{i}\left[1+\frac{v_{i \alpha} u_{\alpha}}{c_{s}^{2}}+\frac{1}{2}\left(\frac{v_{i \alpha} u_{\alpha}}{c_{s}^{2}}\right)^{2}-\frac{u^{2}}{2 c_{s}^{2}}\right] \\
& f_{0}^{(0)}=\rho t_{0}\left(1-\frac{u^{2}}{2 c_{s}^{2}}\right)
\end{aligned}
$$


where the coefficients $t_{i}$ are weights associated with the lattice direction, $c_{s}$ is the speed of sound and $v$ the lattice speed, i.e. $v=\Delta r / \Delta t$ with $\Delta r$ the lattice spacing.

Here we use the so-called $D 2 Q 9$ topology, i.e. a two dimensional square lattice with 9 velocity vectors: directions $i=1,2,3,4$ correspond to the nearest neighbors and directions $i=5,6,7,8$ to the neighbors along the diagonals). For this topology, we take $c_{s}^{2}=1 / 3$ and it is found that the lattice weights are $t_{0}=4 / 9, t_{1,2,3,4}=1 / 9$ and $t_{5,6,7,8}=1 / 36$

Equation (2) together with (4) for the local equilibrium distributions is equivalent to the continuity equation and Navier-Stokes equation with pressure field

$$
p=c_{s}^{2} \rho
$$

and viscosity

$$
\nu=\Delta t c_{s}^{2}\left(\tau-\frac{1}{2}\right)
$$

\section{Simulation of Fluid Flow Past an Impermeable Cylinder}

The main goal of this paper is to compute the drag force $F_{D}$ experienced by a fractal aggregate immersed in a fluid flowing at speed $u_{0}$. We are interested in small Reynolds number regime.

Before we consider this problem, we first revisit a simpler situation, namely the drag force on a cylinder. This is a classical experiment in fluid hydrodynamics. Usually the experimental data give the drag force on a cylinder placed in a system that can be assumed infinite. However, in a numerical simulation, one has to take into account the fact that the computational domain has a finite size and also pay attention to the imperfect discretization of the cylinder on the lattice used for the computation. As these effects will also play a role for the fractal aggregates we want to control them for the cylinder.

We consider a simulation on a periodic lattice of size $N_{x} \times N_{y}$. Since the model is $2 \mathrm{D}$, the cylinder is represented by a disk of diameter $d$, centered at position $\left(N_{x} / 2, N_{y} / 2\right)$. The so-called full-way bounce back rule is applied on the surface of the cylinder, that is, upon collision with the obstacle, the fluid particles simply reverse their direction of motion.

The bounce back rule is particularly easy to implement but it does not ensure a perfect no-slip boundary condition. A way to guarantee a zero velocity on the surface of the obstacle is to use the recently developed mass conserving boundary condition [12] [11].

In our experiments, we accelerate the fluid with a body force $\mathbf{F}=(G, 0)$. In the stationary regime, the amount of momentum injected in the system by this body force should be equal to the momentum adsorbed by the cylinder. The latter is precisely the drag force $F_{D}$. Thus, we must have

$$
F_{G}=\sum_{r_{s}} \sum_{i} 2 f_{i}\left(\mathbf{r}_{s}\right) v_{i}=F_{D}
$$


where $F_{G}$ is total body force added to the system per time step and $\mathbf{r}_{s}$ designate the sites on the cylinder surface. This relation is illustrated in figure 1 .

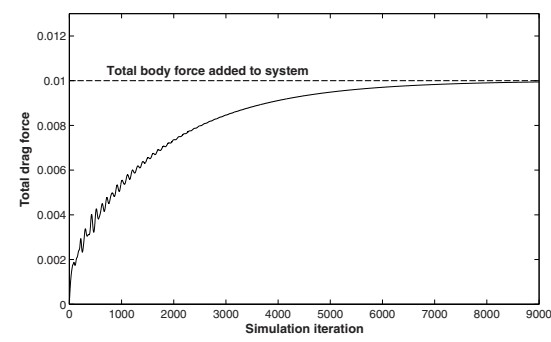

Fig. 1. Development of total drag force on cylinder with an applied body force. We see that in the stationary regime the drag force is equal to the total body force.

Therefore, by applying a body force, one controls the drag force and not the fluid velocity $u_{0}$. This velocity $u_{0}$ should be measured from the simulation. We choose to take the value at location $\left(N_{x}, N_{y} / 2\right)$.

Note that instead of a body force, we could have accelerated the fluid by imposing a velocity profile with $\mathbf{u}_{x}(\mathbf{r})=u_{0}$ at the inlet of the channel. Then the fluid velocity would be the control parameter and the drag force would be measured as the momentum adsorbed per time step at the cylinder surface. We found the second method numerically less accurate.

\subsection{Effect of the Channel Width}

In this section we analyze the behavior of the drag coefficient $C_{D}$ as a function of the Reynolds number of the flow. We consider various channel width $L$ and compare the results of our numerical simulation with the experimental data of Tritton [13]. In this simulation the cylinder radius is always of 8 sites, and the channel length is $N_{x}=N_{y}=L / \Delta r$, where $\Delta r$ is lattice spacing.

Conventionally, the drag coefficient for a cylindrical obstacle is defined as [14]

$$
C_{D}=\frac{F_{D}}{\frac{1}{2} \rho u_{0}^{2} d}
$$

where $d$ is the cylinder diameter and $u_{0}$ is the fluid velocity.

The drag coefficient is often expressed in terms of the Reynolds number

$$
R e=\frac{\rho u_{0} d}{\mu}
$$

with $\mu$ the dynamic viscosity.

It is usually assumed that the relation between $R e$ and $C_{D}$ is

$$
C_{d}=a R e^{-b}
$$


where $a$ and $b$ are constants empirically determined. For a cylinder and for small $R e$ regimes the value of $b$ is found smaller than 1 . The experimental data from Tritton [13] are plotted in a log-log scale in figure 2. We also show the drag coefficients measured in the LB simulations for different channel widths $L=60$, $100,140,180,220$ but the same cylinder radius $d / 2=8$. We see that relation (10) is well obeyed but that the values of $a$ and $b$ depend on $L$.

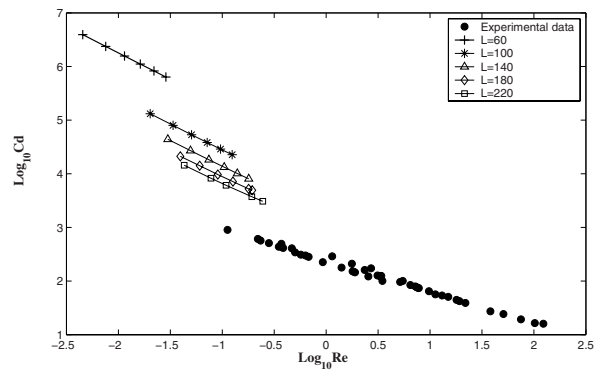

Fig. 2. Plot in log-log scale of Cd vs Re for the experimental data and simulation data.

Tritton's data correspond to an infinitely large channel and are slowly approached by the simulation as $L \rightarrow \infty$. In order to quantify how the drag coefficient in a finite system converges to that of the infinite system, we plot the coefficient $\log a(L)$ and $b(L)$ as a function of $1 / L$.

Figure 3 (left) shows that the vertical offset $\log a(L)$ varies linearly with $1 / L$ and that the limit for $L \rightarrow \infty$ is 2.3534, very much in agreement with the the vertical offset 2.3868 of Tritton's data. A similar fit can be done for $b(L)$, though
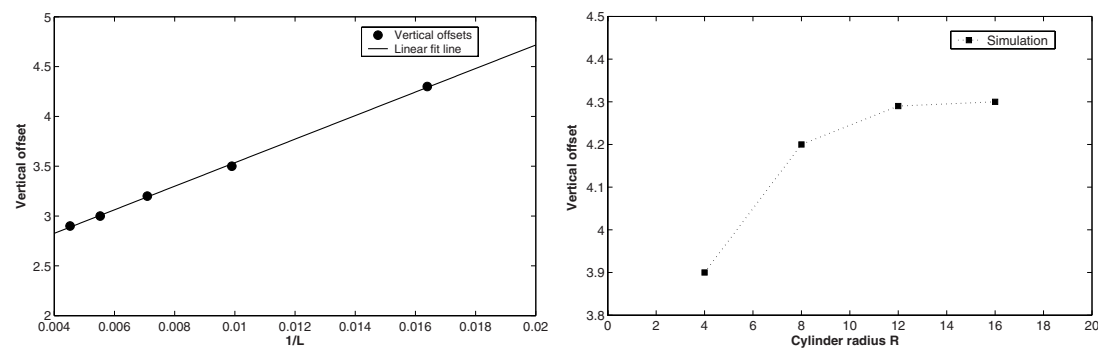

Fig. 3. Vertical offsets versus $1 / L$ and discretization effect

less convincing. As a result, we obtain that the drag coefficient in a channel of width $L$ behaves as

$$
C_{d}=10^{((115.7 / L)+2.35)} R_{e}^{((-7.3 / L)-0.88)}
$$


This rather slow convergence is due to the fact that, at low Reynolds number, the influence of the obstacle on the flow is visible even far away.

\subsection{Discretization Effect}

In the simulation, the cylinder is discretized on the lattice. Therefore its shape is not perfectly circular. However, the bigger the radius the smaller the errors. Here we study how the drag coefficient is sensitive to the discretization errors. We consider several simulations in which we increase in the same ratio both the diameter $d$ of the cylinder and the system size $L$. The scaling factors we use are $0.5,1,1.5$, and 2. For each of them we compute the drag coefficient at $R e=1$

The results are shown in figure 3 (right). We observe that when the radius is above 16 lattice site, the drag coefficient stabilizes. Between the value for $d / 2=8$ and $d / 2>16$, there is an error of about $10 \%$.

\subsection{Drag as a Function of the Volume Fraction}

The situation of an infinite array of cylinders subject to a flow at speed $u_{0}$ has been investigated by Sangani and Acrivos [15]. A semi-analytical solution connecting the drag, fluid velocity and solid volume fraction has been proposed. This situation corresponds exactly to our numerical simulation, since our boundary conditions are periodic. The relation considered in [15] is the following

$$
\frac{F_{D}}{\mu U}= \begin{cases}4 \pi /\left\{\ln \left(c^{-1 / 2}\right)-0.738+c-0.887 c^{2}+2.038 c^{3}\right\} & \text { if } c \ll 1 \\ 9 \pi / 2 \sqrt{2}\left\{1-\left(\frac{c}{c_{\max }}\right)^{1 / 2}\right\}^{-5 / 2} & c_{\max }-c \ll 1\end{cases}
$$

where $F_{D}$ is total drag, $\mu$ is dynamic viscosity of fluid and $U$ is known as the average velocity of flow. The quantity $c$ is the solid volume fraction (i.e the ratio of the cylinders area to the system area) and $c_{\max }$ is volume fraction obtained when the cylinders are large enough to touch each other.

Figure 4 compares the above theoretical expression (solid line) with our numerical simulations (circles and squares). Whereas the agreement is good in the limit of small $c$, the numerical experiment departs from the behavior predicted by eq. (12). This discrepancy is not due to the slip velocity resulting from the bounce back rule since the mass conserving boundary condition does not change the result in a significant way.

A reason that can yet explain this difference of behavior is the definition of $U$. This average velocity is not precisely defined in [15]. In our simulation we computed it as $U=\sum_{\mathbf{r}} u_{x}(\mathbf{r}) / L^{2}$. In the figure, we also show the curves we obtain if $U$ is taken as the minimal or maximal velocity in the fluid. Clearly both the numerical and analytical results lie within these two extreme limits.

\section{Hydrodynamic Properties of Fractal Aggregates in 2D}

In this section we consider a fractal obstacle in the flow instead of a cylinder. We want to investigate the effect of the fractal geometry on the drag force. 


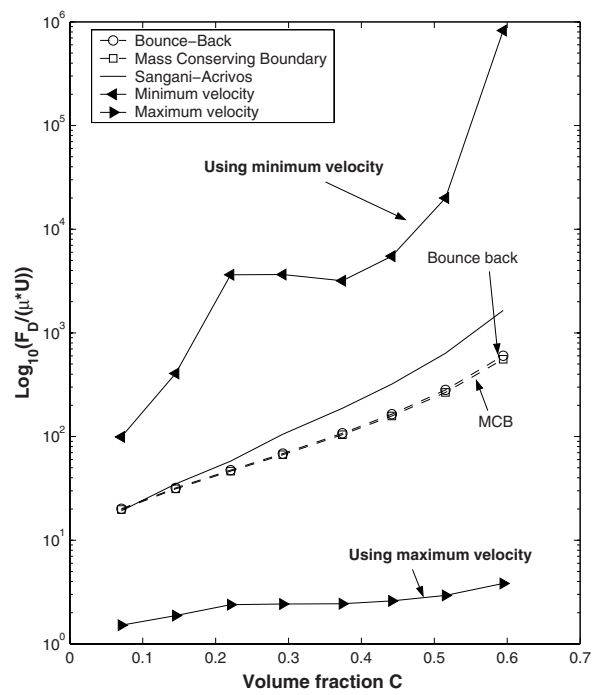

Fig. 4. Drag force as a function of the volume fraction c. The graph compares theory with LB simulations using either bounce back or mass conserving boundary conditions. The observed discrepancy is not yet understood.

In our simulations, fractal aggregates are composed of many elementary particles located on the lattice sites. They are obtained by simulating a lattice DLA (diffusion limited aggregation) process.

In the preliminary experiments described in this paper, we consider rather small fractal objects, i.e. aggregates having 100 or 200 particles. For the sake of simplicity, we also assume that the particles making up the fractal are point-like and occupy only one lattice site. Figure 5 shows the 100-particle fractal and its effect on the velocity of fluid. We proceed our investigations by comparing the drag force experienced by the aggregate as a function of its orientation with the main flow. We consider only four orientations, namely the reference position, and the rotation by $\pi / 2, \pi$ and $3 \pi / 2$.

As before, we impose the drag force $F_{D}$ on the system by applying a body force on the fluid and we measure the resulting average flow velocity $u$ (which is computed by averaging velocity over all fluid sites) of the flow in the system. Figure 6 (left) shows that the relation between $F_{D}$ and $u$ is linear, as expected from Stokes law, but that the proportionality coefficient $F_{D} / u$ depends on the orientation of the fractal object. In addition, we observe that the behavior is the same whether the object is rotated by $180^{\circ}$ or not.

These results could suggest that the hydrodynamic resistance of the fractal object to the fluid motion mostly depends on its cross section orthogonal to the flow direction. 

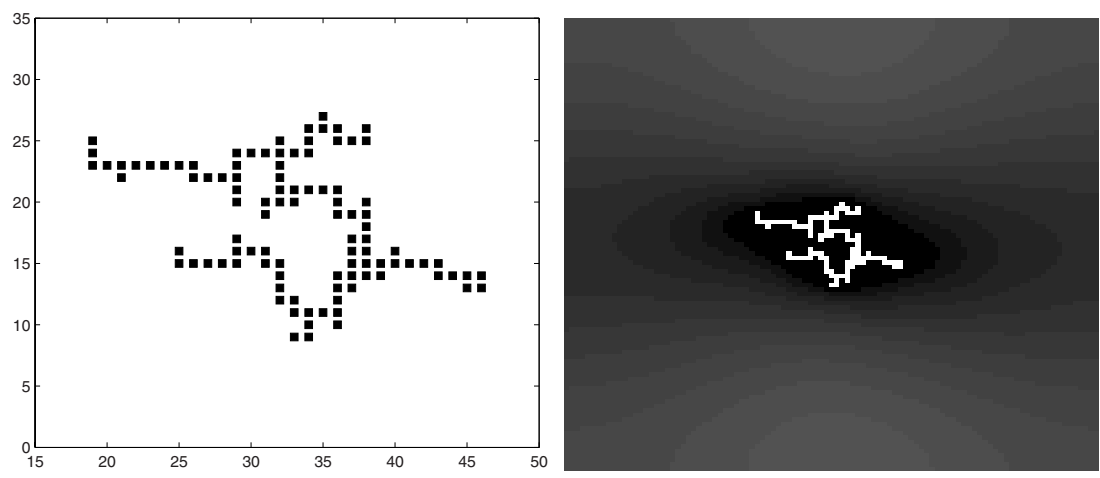

Fig. 5. Fractal aggregate used for the simulation and the resulting intensity of the flow speed

However, as shown in figure 6 (right) results from simulations with a plate orthogonal to the flow direction and having the same width as the projection of the fractal aggregate do not show the same behavior. A plate offers less resistance to the flow than the fractal object. This is due to the fact that the length of the obstacle along the flow direction also plays a role, yet to a lesser extent than the perpendicular extension. Therefore, we also compare the drag force on rectangular objects having the same length and width as the fractal object. The plots shown in figure 6 (right) indicate that the rectangle now over-estimate the drag force.

Nevertheless, it clearly appears from the simulation that the speed of the fluid inside the aggregate is almost zero. Therefore, in $2 \mathrm{D}$, the hydrodynamic properties of a fractal object are mostly determined by its outer shape and not distribution of particles inside the fractal. We believe that, in $2 \mathrm{D}$, the convex hull of the fractal object would give a good approximation of the hydrodynamic behavior. This observation is also consistent with the results by A.Adrover and M.Giona [7].
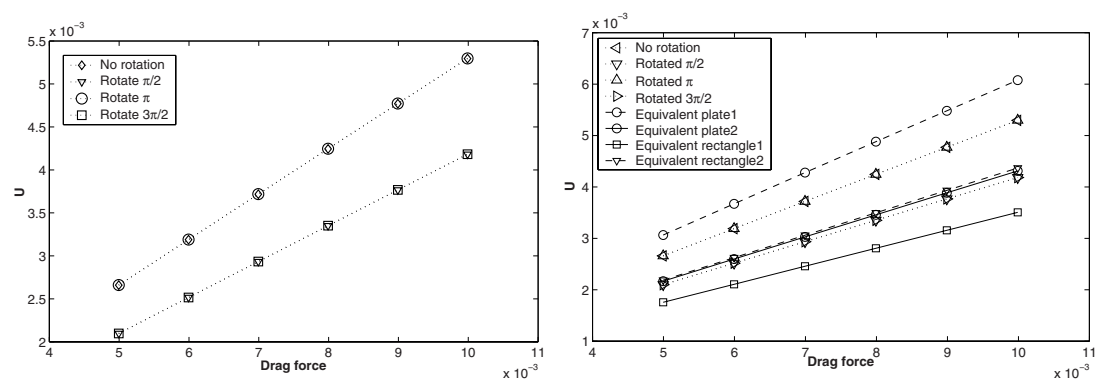

Fig. 6. Velocity $u$ versus the drag force $F_{D}$ for different orientation of the fractal aggregate and various $2 \mathrm{D}$ objects. 
For 3D fractal objects, hydrodynamic properties are often described in terms of the hydrodynamic radius $R_{h}=F_{D} /\left(6 \pi \mu u_{0}\right)$ and the relation it bears with the gyration radius $R_{g}{ }^{2}=(1 / N) \sum_{i}\left(\mathbf{r}_{i}-\mathbf{r}_{c m}\right)^{2}$, where $N$ is the number of particles in the aggregate, $\mathbf{r}_{i}$ their location and $\mathbf{r}_{c m}$, the center of mass of the object. In $2 \mathrm{D}$, the radius of gyration can be computed in the same way as in $3 \mathrm{D}$. The hydrodynamic radius is given by the radius of a cylinder experiencing the same drag force in a flow with the same speed. From the simulation, we search for the radius $R_{h}$ of a cylinder offering the same resistance as the fractal. This value $R_{h}$ may depend on the chosen orientation of the fractal. For the 0 -rotated and $\pi / 2$ rotated orientation of the 100-particle fractal, we find $R_{h} / R_{g} 1.288$ and 1.675 respectively.

Finally, we consider another DLA fractal object with 200 particles and much more isotropic than the one in figure 5 . In figure 7 we see that for all the four considered orientations, the $F_{D}-u$ relation is the same. It also corresponds rather well to the drag on the cylinder which circumscribes the aggregate. On the other hand, a cylinder whose radius is equal to the gyration radius of the fractal experiences a drag force significantly too small.

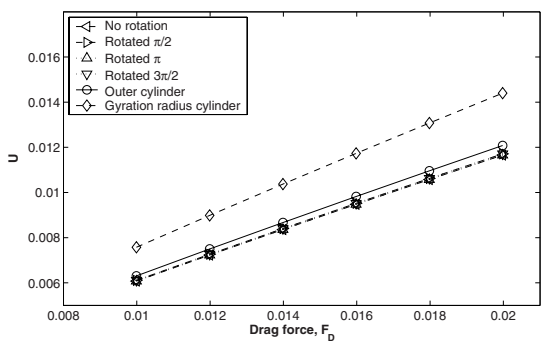

Fig. 7. Average velocity versus drag force for the 200-particle DLA fractal. The hydrodynamic behavior for all orientations and the one (solid line) of the circumscribing cylinder are similar. The upper dashed line is that obtained for a cylinder whose radius is the gyration radius of the fractal

\section{Conclusion}

The Lattice Boltzmann method is a powerful approach for modeling flow and transport at intermediate scales where neither continuum models nor molecular dynamics methods are practical. We have considered this approach to study, for small Reynolds number flows, the drag force acting on a cylinder and on fractal aggregates.

In the case of the cylinder we have been able to estimate the finite size corrections by deriving a scaling law for the drag coefficient as a function of the Reynolds number and system size. We also studied the discretization effect of 
the cylinder on the lattice and we found that, for a radius larger than 16 sites, the error is small.

In the case of a fractal aggregate, we studied the drag force for various orientation with respect to the flow direction. We can conclude from the simulation that the outer shape of the aggregate is the main factor determining the hydrodynamic properties in 2D. The internal structure plays an almost irrelevant role as the fluid is at rest inside the fractal. Thus we do not observe a relation between the fractal dimension and the hydrodynamic radius. This result has never been clearly reported in previous studies. In 3D, however we expect another behavior as the porosity of the aggregate will be crucial. This will be studied in a forthcoming paper.

\section{References}

1. J.G. Kirkwood and J. Riseman. The intrinsic viscosities and diffusion constants of flexible macromolecules in solution. J. Chem. Phys, 16:565-573, 1948.

2. Z.Y. Chen P. Meakin and J.M. Deutch. Comment on hydrodynamic behaviour of fractal aggregates. Physical Review Letters, 59(18):2121, 1987.

3. H.L. Frisch W. Hess and R.Klein. On the hydrodynamic behaviour of colloidal aggregates. Z.Phys.B - Condensed Matter, 64:65-67, 1986.

4. R. Thouy D.Coelho, J-F. Thovert and P. Adler. Hydrodynamic drag and electrophoresis of suspensions of fractal aggregates. Fractals, 5(3):507-522, 1997.

5. B.U. Felderhof B.Cichocki and K. Hinsen. Friction and mobility of many spheres in stokes flow. J. Chem. Phys, 100(5):3780-3790, 1994.

6. Pierre M. Adler. Hydrodynamic properties of fractal flocs. Faraday Discuss. Chem. Soc, 83:145-152, 1987.

7. A.Adrover and M.Giona. Hydrodynamic properties of fractals: application of the lattice boltzmann equation to transverse flow past an array of fractal objects. Int.J.Multiphase Flow, 23(1):25-35, 1997.

8. S. Succi. The Lattice Boltzmann Equation for Fluid Dynamics and Beyond. Oxford University Press, 2001.

9. B. Chopard and M. Droz. Cellular Automata Modeling of Physical Systems. Cambridge University Press, 1998.

10. B. Chopard, A. Dupuis, P. Luthi, and A. Masselot. Cellular automata and lattice boltzmann techniques: An approach to model and simulate complex systems. Advances in Complex Systems, 5:103-246, 2002.

11. A. Dupuis. From a Lattice Boltzmann model to a parallel and reusable implementation of a virtual river. $\mathrm{PhD}$ thesis, University of Geneva, 2002.

12. B. Chopard and A. Dupuis A mass conserving boundary condition for lattice Boltzmann models, (DSFS 2002 conference) submitted to Int. J. Mod. Phys.B, 2002

13. D.J. Tritton. Experiments on flow past a circular cylinder at low reynolds numbers. J. Fluid. Mech, 6:547-567, 1959.

14. D. J. Tritton. Physical Fluid Dynamics. Oxford Science Publications, 1988.

15. A.S.Sangani and A.Acrivos. Slow flow past periodic arrays of cylinders with application to heat transfer. Int.J.Multiphase Flow, 8(3):25-35, 1982.

16. A.J.C. Ladd and R. Verberg. Lattice-Boltzmann simulations of particle fluid suspensions. J. Stat. Phys.,104:1191-1251, 2001. 